\title{
RF power transfer efficiency of inductively coupled low pressure $\mathrm{H}_{2}$ and $\mathrm{D}_{2}$ discharges
}

\author{
D Rauner ${ }^{1,2}, \mathbf{S}$ Briefi $^{1}, \mathbf{U}_{\text {Fantz }}{ }^{1,2}$ \\ ${ }^{1}$ Max-Planck-Institut für Plasmaphysik, Boltzmannstrasse 2, 85748 Garching, \\ Germany \\ ${ }^{2}$ AG Experimentelle Plasmaphysik, Universität Augsburg, 86135 Augsburg, \\ Germany \\ E-mail: david.rauner@ipp.mpg.de
}

May 2017

\begin{abstract}
.
The RF power transfer efficiency and the relevant power absorption mechanisms of inductively heated hydrogen and deuterium plasmas are investigated in the low-pressure region between 0.25 and $10 \mathrm{~Pa}$. The discharges are generated in a cylindrical vessel via a helical coil applying a frequency of $1 \mathrm{MHz}$ and delivered RF powers up to $800 \mathrm{~W}$. The power transfer efficiency $\eta$ is quantified by a subtractive method that relies on the measurement of the delivered RF power and of the RF current through the plasma coil both with and without discharge operation. By means of optical emission spectroscopy and electrical probe measurements, the key plasma parameters are obtained. For both $\mathrm{H}_{2}$ and $\mathrm{D}_{2}$, the relative behaviour of the power transfer efficiency is well comparable, which increases with increasing delivered RF power and describes a maximum at pressures between 1 and $3 \mathrm{~Pa}$ where more than $90 \%$ of the provided power are absorbed by the plasma. The observed relative dependencies of $\eta$ on the operational parameters are found to be well explained by an analytical approach that considers the power absorption by the plasma via evaluating the RF plasma conductivity based on the measured plasma parameters. At the parameters present, non-collisional stochastic heating of electrons has to be considered for pressures $p \leq 1 \mathrm{~Pa}$, while collisional heating dominates at higher pressure. Molecular dissociation is found to have a significant influence on the power transfer efficiency of light molecular discharges. The direct comparison of $\mathrm{H}_{2}$ and $\mathrm{D}_{2}$ identifies the higher atomic density in deuterium to cause a systematically increased power transfer efficiency due to an increased ionization rate in the present electron temperature region.
\end{abstract}

Keywords: radio frequency discharge, inductively coupled plasma, power transfer efficiency, electron heating, hydrogen, deuterium, molecular dissociation 


\section{Introduction}

Inductively coupled plasmas (ICPs) are one of the most important and widespread implementations of radio frequency $(\mathrm{RF})$ driven low-pressure discharges. Their importance for both industrial as well as scientific applications relies on their capability to achieve high densities even for comparably low input RF powers, as well as on the possibility of controlling the ion energies at the plasma boundaries independently.

Within the last decades, significant experimental and theoretical efforts regarding the understanding of ICPs and the respective plasma heating mechanisms have been made in order to optimize the discharge properties according to the individual requirements at hand $[1,2,3]$. Of particular interest has been the investigation and improvement of the RF power transfer efficiency, which describes how much of the electrically provided RF power is actually absorbed by the plasma. In opposition to the simple assumption that the RF power delivered by the generator equals the power absorbed by the plasma, it has been conclusively shown that transmission power losses can in fact be substantial $[4,5,6,7,8]$. The occurring losses originate from ohmic heating in the electrical transmission, the matching network and the plasma coil as well as from induced eddy currents within conducting material near the RF system.

The RF power transfer efficiency, or analogously the plasma equivalent resistance, is known to be influenced by a variety of external factors such as the applied frequency, the specific experiment geometry, the gas type or the operational parameters of gas pressure and delivered RF power. In order to understand the behaviour of power coupling to an inductive RF discharge, the electron heating mechanisms of inductively coupled plasmas have been closely studied. In addition to classical collisional (ohmic) heating, the influence of non-collisional stochastic heating has been found to play a substantial role at low pressure and low excitation frequency $[2,9,10,11]$.

The reported investigations on the $\mathrm{RF}$ power transfer efficiency have been performed almost exclusively in noble gas discharges, and mainly in Argon. However, the importance of inductive RF discharges utilizing light molecular gases like hydrogen and deuterium has grown over the last years. Beside the important role of hydrogen plasmas for material treatment purposes, $\mathrm{H}_{2}$ and $\mathrm{D}_{2}$ discharges are used in $\mathrm{RF}$ driven ion sources of particle accelerators and the neutral beam heating systems for fusion $[12,13]$. In contrast to noble gas discharges, the additional processes of rotational and vibrational excitation as well as molecular dissociation can significantly influence the power deposition in molecular discharges. A fundamental investi- gation of the power absorption processes in inductively driven discharges in light molecular gases has not been reported up to now to the author's knowledge.

The RF power transfer efficiency and the RF power absorption in inductive hydrogen and deuterium discharges is systematically investigated in the present work, focussing on the low frequency and lowpressure regime. The measurements are performed in continuous wave operation at an experimental setup utilizing a cylindrical discharge vessel, a helical plasma coil and an RF generator operating at an excitation frequency of $1 \mathrm{MHz}$. Measurements are performed at varying gas pressure between 0.3 and $10 \mathrm{~Pa}$ and delivered RF powers up to $800 \mathrm{~W}$. The RF power transfer efficiency $\eta$ is quantified by a subtractive method which relies on the determination of the power absorbed by the plasma from the difference between the delivered RF power with a discharge and the power delivered without a discharge $[5,14,15]$. In order to gain detailed insight into the power absorption mechanism, the key plasma parameters are measured by means of optical emission spectroscopy and electrical probe measurements. For hydrogen discharges, the dependence of the power absorption on the gas pressure and the applied $\mathrm{RF}$ power is investigated by a simplified analytical approach, capable of considering both collisional and non-collisional electron heating mechanisms. The importance of considering the latter for the present operational regime is addressed. In order to discuss the influence of the different neutral species (atoms and molecules) in light molecular plasmas on the power transfer efficiency, inductive discharges in hydrogen and deuterium are compared. This comparison is suitable, since the difference in mass does generally not influence the electronic, but the vibrational and rotational states of the molecules. This affects especially the dissociation processes, leading to a typically higher atomic density in low pressure $\mathrm{D}_{2}$ discharges in comparison to $\mathrm{H}_{2}$ at the same operational parameters.

\section{Power absorption mechanism of inductively coupled plasmas}

In inductive discharges, the power transfer from the electric field to the plasma electrons occurs within the skin depth layer at the plasma surface. If the collision frequency of electrons with neutrals $\nu_{\mathrm{en}}$ is much larger than the applied frequency $\omega$, the dominant heating mechanism is collisional (ohmic) power dissipation. If the plasma is treated as a conductor, the RF power absorption by the plasma is given by [2]

$P_{\text {plasma }}=\frac{1}{2} \operatorname{Re}\left(\sigma_{\mathrm{p}}\right)|\tilde{\mathbf{E}}|^{2}$, 
where $\tilde{\mathbf{E}}$ denotes the complex electric field amplitude and $\sigma_{\mathrm{p}}$ the complex plasma conductivity. The real part of $\sigma_{\mathrm{p}}$ is given by

$\operatorname{Re}\left(\sigma_{\mathrm{p}}\right)=\operatorname{Re}\left(\frac{n_{\mathrm{e}} e^{2}}{m_{\mathrm{e}}\left(i \omega+\nu_{\mathrm{en}}\right)}\right)=\frac{n_{\mathrm{e}} e^{2}}{m_{\mathrm{e}}} \frac{\nu_{\mathrm{en}}}{\omega^{2}+\nu_{\mathrm{en}}^{2}}$.

The average frequency of collisions of electrons with neutral particles can be estimated by the product of the neutral particle density $n_{0}$ with the rate coefficient $X_{\mathrm{en}}\left(T_{\mathrm{e}}\right)$ of the momentum transfer collisions of electrons [2]. The latter is determined by integrating over the product of a Maxwellian electron energy distribution function (EEDF) and the relevant electron momentum transfer cross section data, which are in case of hydrogen and deuterium for example provided by Yoon et al. $[16,17]$.

It is well known however, that in the low-pressure limit, where the collision frequency of electrons with neutrals is of the same order (or smaller) than the excitation frequency, non-collisional stochastic heating of electrons plays a dominant role $[2,9,18]$. This non-local kinetic effect occurs, when the transit time of electrons in the RF sheath is much shorter than the RF period. In that case, electrons can acquire a net velocity change and are adiabatically heated. The stochastic heating in inductive discharges can be accounted for in analogy to pure collisional heating by defining the stochastic collision frequency $\nu_{\text {stoc }}$.

The stochastic collision frequency can be calculated following the approach by [10]. For the present case, the excitation frequency $\omega$ is always much lower than the thermal transit frequency of electrons through the RF sheath. Therefore the stochastic collision frequency is determined by

$\nu_{\text {stoc }}=\frac{1}{2 \pi} \frac{\bar{v}_{\mathrm{e}}}{\delta}$,

with the Maxwellian mean velocity of electrons $\bar{v}_{\mathrm{e}}$ and the RF skin sheath depth $\delta$. In the present case, the latter is obtained from

$\delta=\left(\frac{c^{2}}{\omega_{\mathrm{pe}}^{2}} \frac{\bar{v}_{\mathrm{e}}}{\pi \omega}\right)^{1 / 3}$,

where $\omega_{\text {pe }}$ is the electron plasma frequency.

In order to account for both collisional as well as non-collisional heating of electrons, an effective collision frequency $\nu_{\mathrm{eff}}=\nu_{\mathrm{en}}+\nu_{\mathrm{stoc}}$ is defined. The validity of this assumption which considers both local and non-local heating mechanisms in inductive discharges over a broad collisionality regime has been conclusively shown $[2,10]$. Consecutively, the plasma conductivity can then be written as

$\operatorname{Re}\left(\sigma_{\mathrm{p}}\right)=\frac{n_{\mathrm{e}} e^{2}}{m_{\mathrm{e}}} \frac{\nu_{\mathrm{eff}}}{\omega^{2}+\nu_{\mathrm{eff}}^{2}} \equiv \sigma_{\mathrm{eff}}$.
According to Equations (1) and (5) the power absorption of the plasma is - in a simplified 0dimensional consideration neglecting the influence of the electric field $\tilde{\mathbf{E}}$ as well as spacial variations of the involved plasma parameters - proportional to $n_{\mathrm{e}}$ and the fraction $\nu_{\text {eff }} /\left(\omega^{2}+\nu_{\text {eff }}^{2}\right)$, that reaches its maximum at $\nu_{\text {eff }}=\omega$.

Apart from the spatial simplifications of this description, there is an additional limitation if the heating of $\mathrm{RF}$ driven hydrogen discharges at low frequency is analyzed. Due to the low ion mass, the ion plasma frequency can be significantly higher than the excitation frequency. This is relevant especially for the definition of stochastic heating, as it contradicts the assumption that the ions do not respond to the RF fields, which is used to derive the equations (3) and (4) [10]. This restriction has to be considered during the discussion of the plasma heating and power transfer processes at low pressure and is further assessed in the discussions of section 4.1 .

\section{Experimental setup and diagnostics}

A sketch of the experimental setup and the applied diagnostic systems is shown in Figure 1. The cylindrical discharge vessel is made out of quartz glass with a length of $40 \mathrm{~cm}$, an outer diameter of $10 \mathrm{~cm}$ and a wall thickness of $5 \mathrm{~mm}$. At the ends of the cylinder the vacuum system is attached to the gas feed line on one side and the pumping system on the other side. The setup allows for continuous wave plasma operation at gas pressures between 0.25 and $10 \mathrm{~Pa}$ in hydrogen and deuterium at a fixed gas flow of $5 \mathrm{sccm}$. The absolute gas pressure in the vessel is adjusted by a valve reducing the pumping rate and measured by a capacitance manometer.

Around the center of the discharge vessel the helical coil for inductive plasma operation is installed, consisting of five windings of copper tubes $(6 \mathrm{~mm}$ diameter) with a measured total inductance of $2.2 \mu \mathrm{H}$. Via a $\gamma$-topology matching network, the antenna is connected to the RF generator operating at the excitation frequency of $1 \mathrm{MHz}$ with a maximum output power of $1 \mathrm{~kW}$. The capacitors of the matching network are adjustable, allowing to tune $\mathrm{C}_{1}$ between 0.5 and $2 \mathrm{nF}$ and $\mathrm{C}_{2}$ between 9 and $10.5 \mathrm{nF}$. Thereby, impedance matching to the generator output resistance of $50 \Omega$ for both hydrogen and deuterium plasma operation is possible within the whole pressure range of interest, allowing the generator to deliver the set output power to the respective load. For the presented investigations, no Faraday shield is applied. Therefore capacitive coupling is not generally suppressed, even though the efficiency of capacitive discharges at the low excitation frequency of $1 \mathrm{MHz}$ is very weak. In 


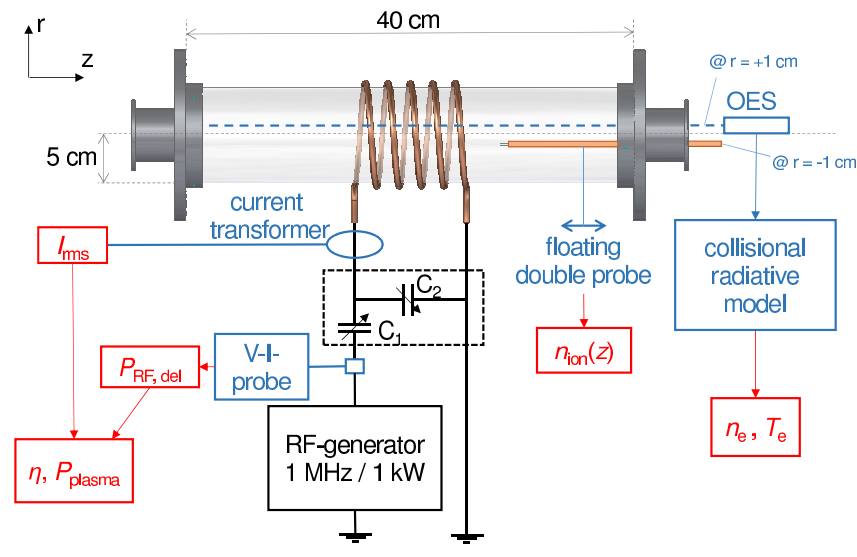

Figure 1. Scheme of the experimental setup and the applied diagnostic systems.

order to ensure the plasma is driven inductively, the operational parameters of all discharges investigated are chosen accordingly, foremost by applying $\mathrm{RF}$ powers sufficiently high to suppress the occurrence of E- to H-mode transitions within the whole parameter range.

The impedance matching of the discharges is monitored in-situ by an in-line voltage and current probe that is installed between the generator and the matching circuit. Additionally, this probe is able to measure the value of the delivered $\mathrm{RF}$ power $P_{\mathrm{RF}}$,del precisely. By means of a current transformer, which is located around the connection of the coil to the matching network, the RF current running through the coil is measured. The combined measurement of the antenna current and the totally delivered RF power also allows for the required quantification of the $\mathrm{RF}$ power transfer efficiency $\eta$.

The applied method relies on the measurement of the power losses $P_{\text {network }}$ during plasma operation due to ohmic heating, occurring both within the RF coil and the matching network as well as via induced eddy currents in the vicinity of the RF system. In order to quantify these losses, the real part of the complex resistance of the $\mathrm{RF}$ coil and the matching network $R_{\text {network }}$ is measured in pure vacuum operation. The measurement procedure that is applied is described in detail by [5]. With $P_{\text {plasma standing for the power }}$ that is actually absorbed by the plasma, the RF power transfer efficiency is calculated according to

$\eta=\frac{P_{\text {plasma }}}{P_{\mathrm{RF}, \mathrm{del}}}=\frac{P_{\mathrm{RF}, \mathrm{del}}-I_{\mathrm{rms}}^{2} R_{\text {network }}}{P_{\mathrm{RF}, \mathrm{del}}}$.

During the investigations presented here, the network and coil resistance $R_{\text {network }}$ is typically around $0.1 \Omega$. The antenna current $I_{\text {rms }}$ varies within the range of 25 to $50 \mathrm{~A}$ during plasma operation, strongly depending on the gas pressure and the delivered RF power. In principle, the applied method provides a lower estimate of the RF power transfer efficiency as it does not account for the screening of electromagnetic fields by the plasma and thereby tends to overestimate the losses via induced eddy currents [5]. However, at the presented experimental setup, this is assumed to have little influence, as there are no conducting elements in the direct vicinity of the RF coil. The nearest conductors are the metallic end plates which are mounted on either side of the vessel in a distance of roughly $15 \mathrm{~cm}$ to the $\mathrm{RF}$ coil.

In order to obtain the required plasma parameters, both quantitative optical emission spectroscopy (OES) and electrical probe measurements have been used. The measurement positions of both diagnostic systems are located in the same radial distance of $1 \mathrm{~cm}$ to the axis of the cylinder in direct opposition to each other. This allows for a direct comparison, as the discharge is assumed cylindrically symmetric.

The spectroscopic measurements have been carried out with an intensity calibrated high-resolution spectrometer $\left(\Delta \lambda_{\mathrm{FWHM}} \approx 18 \mathrm{pm}\right)$ along a line of sight parallel to the cylinder axis. Thereby, the absolute emissivities of the atomic Balmer lines from $\mathrm{H}_{\alpha}$ to $\mathrm{H}_{\delta}$ (analogously in deuterium from $\mathrm{D}_{\alpha}$ to $\mathrm{D}_{\delta}$ ) and the molecular Fulcher transition $\left(\mathrm{d}^{3} \Pi_{\mathrm{u}} \rightarrow \mathrm{a}^{3} \Sigma_{g}^{+}\right.$, located between 590 and $650 \mathrm{~nm}$ ) are detected. In order to evaluate the measured data, the collisional radiative models Yacora $\mathrm{H}$ and Yacora $\mathrm{H}_{2}[19,20]$ are applied. The zero dimensional models balance the relevant population and depopulation processes of the considered states of $\mathrm{H}$ and $\mathrm{H}_{2}$, yielding population densities. For the presented evaluations, the plasma was considered to be optically thin. By evaluating the intensity ratio of the atomic $\mathrm{H}_{\gamma}\left(\mathrm{D}_{\gamma}\right)$ line to the molecular Fulcher band emission, the atomic to molecular density ratio is determined [21]. The electron density and temperature are obtained from the models via a fitting procedure, where $n_{\mathrm{e}}$ and $T_{\mathrm{e}}$ are varied until both the emissivities as well as the line intensity ratios of the measurement are matched.

Furthermore, the evaluation of the Fulcher system allows for a determination of the gas temperature $T_{\text {gas }}$ for both hydrogen and deuterium. The exact procedure applied here to obtain $T_{\text {gas }}$ follows the method described in detail in [22]. Taking account of the measured pressure and gas temperature during plasma operation, the neutral particle density $n_{0}$ is subsequently determined according to the ideal gas law. All parameters determined via OES have to be considered as average values along the line of sight.

In addition to the spectroscopic investigations, a movable floating double probe is applied [23, 24]. It allows for the local determination of the positive ion density $n_{\text {ion }}$ and, considering quasineutrality, of the 
electron density $n_{\mathrm{e}}=n_{\text {ion }}$. The probe consists of two parallel identical tungsten wires with a diameter of $300 \mu \mathrm{m}$ and a length of $1 \mathrm{~cm}$, installed in a distance of $0.5 \mathrm{~cm}$ to each other. A classical single probe cannot be used in this experiment due to the lack of conducting walls within the dielectric discharge tube that would serve as the grounded reference potential. The position of the double probe can be varied parallel to the axis within a range of $20 \mathrm{~cm}$. This allows for space resolved measurements of $n_{\mathrm{e}}$ between the discharge centre and the end plates. In order to compare the space resolved data of $n_{\mathrm{e}}$ with the LOSaveraged spectroscopic investigations, the probe data is axially averaged.

\section{Results and discussion}

\subsection{RF power transfer efficiency in $\mathrm{H}_{2}$}

In Figure 2 (a), the RF power transfer efficiency obtained in inductively driven hydrogen discharges is shown at varying gas pressure between 0.3 and $10 \mathrm{~Pa}$. Presented are measurements obtained at delivered RF powers of $250 \mathrm{~W}, 520 \mathrm{~W}$ and $800 \mathrm{~W}$, respectively. With increasing the totally delivered RF power, the efficiency increases systematically, whereas a broad maximum of $\eta$ over pressure appears between 1 and $3 \mathrm{~Pa}$. In this pressure region, the $\mathrm{RF}$ power transfer efficiency exceeds values of $90 \%$ for $\mathrm{RF}$ powers of $800 \mathrm{~W}$. At the low and high pressure limits, the efficiency drops significantly down to values below $50 \%$, particularly for low RF power. At $250 \mathrm{~W}$ delivered RF power, the stable ignition and sustainment of an inductive discharge at the low and high pressure limits of 0.3 and $10 \mathrm{~Pa}$ is no longer possible due to the decreased power absorption by the plasma. With increasing power, also the position of the peak slightly shifts from $1 \mathrm{~Pa}$ at $250 \mathrm{~W}$ to $3 \mathrm{~Pa}$ at $800 \mathrm{~W}$. Generally, the observed dependence of $\eta$ on $p$ and $P_{\mathrm{RF} \text {,del }}$ is mainly caused by a change of the antenna current $I_{\text {rms }}$ which is illustrated in Figure 2 (b) for the delivered RF power of $520 \mathrm{~W}$. It is of a value around $28 \mathrm{~A}$ in the pressure region between 1 and $3 \mathrm{~Pa}$ and significantly rises towards higher and lower pressure up to values of $50 \mathrm{~A}$. As the network resistance is of roughly $0.1 \Omega$ virtually independent of the operational parameters, the relative behaviour of the loss term $I_{\text {rms }}^{2} R_{\text {network }}$ in Equation (6) is dominated by the change of the antenna current, which subsequently leads to the observed behaviour of $\eta$. The pressure dependent change of the current is a result of the varying power absorption by the plasma, which acts like an additional resistance within the RF network, commonly defined as the plasma equivalent resistance. The observed behaviour is in general well comparable to the results of the power transfer efficiency of noble gas discharges that have been reported several times, e.g. by $[5,8,25]$.

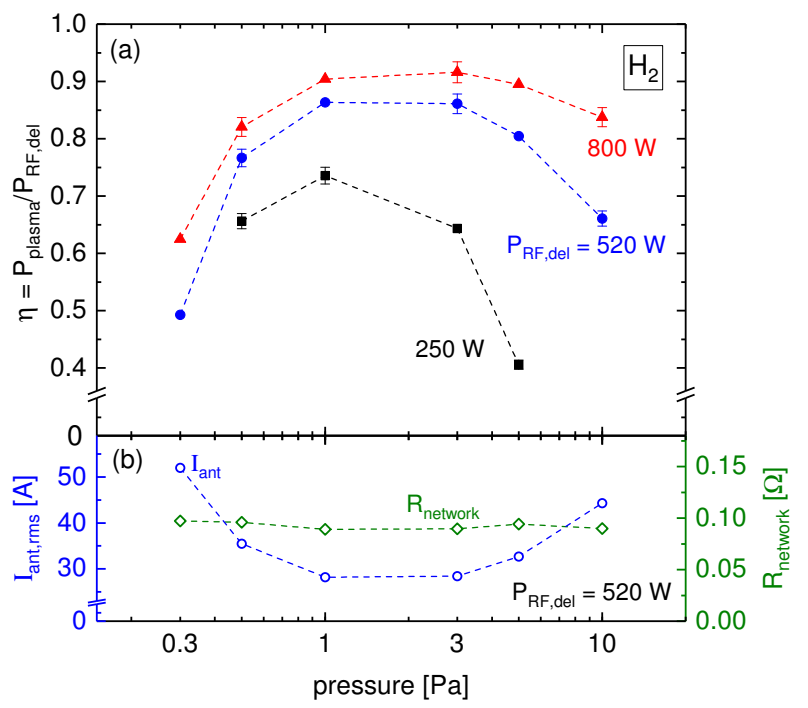

Figure 2. (a) RF power transfer efficiency $\eta$ in hydrogen discharges at varying pressure and different values of the delivered RF power $P_{\mathrm{RF} \text {, del }}$. In (b) the the antenna current during plasma operation and the network resistance at $520 \mathrm{~W}$ are depicted.

The results presented in Figure 2 are further examined by the analytical considerations described in section 2, thereby taking into account the measured plasma parameters and the relevant electron heating mechanisms. In order to analyse the pressure and power dependence of the RF power transfer efficiency $\eta$, the experimentally obtained parameters $n_{\mathrm{e}}, T_{\mathrm{e}}$ and $\nu_{\text {eff }}$ are discussed in the following, which determine the plasma conductivity $\sigma_{\text {eff }}$ defined in Equation (5), and consecutively the power absorption by the plasma according to Equation (1).

Presented in Figure 3 are the electron density $n_{\mathrm{e}}$ and the electron temperature $T_{\mathrm{e}}$ at varying pressure and delivered RF power. In (a), the axial average of the electron density for delivered RF powers of 250,520 and $800 \mathrm{~W}$ is displayed as determined by the floating double probe. $n_{\mathrm{e}}$ is of the order of $10^{17} \mathrm{~m}^{-3}$, increases with increasing $\mathrm{RF}$ power and - in the lower pressure region up to $3 \mathrm{~Pa}$ - also with pressure. In the region above $3 \mathrm{~Pa}$, a different behaviour depending on the delivered power is observed. For $800 \mathrm{~W}$, the density continues to grow, while for intermediate and lower power it saturates or even decreases in the high pressure region, which is connected to the decrease of the RF power transfer efficiency. In addition to the probe data, the electron density obtained via OES is exemplarily shown for $P_{\mathrm{RF} \text {,del. }}=$ $520 \mathrm{~W}$. The correspondence to the electron density 
obtained by the floating double probe is excellent, regarding both the absolute value and the relative trend, which demonstrates the reliability of the density measurements and the cylindrical symmetry of the discharge.
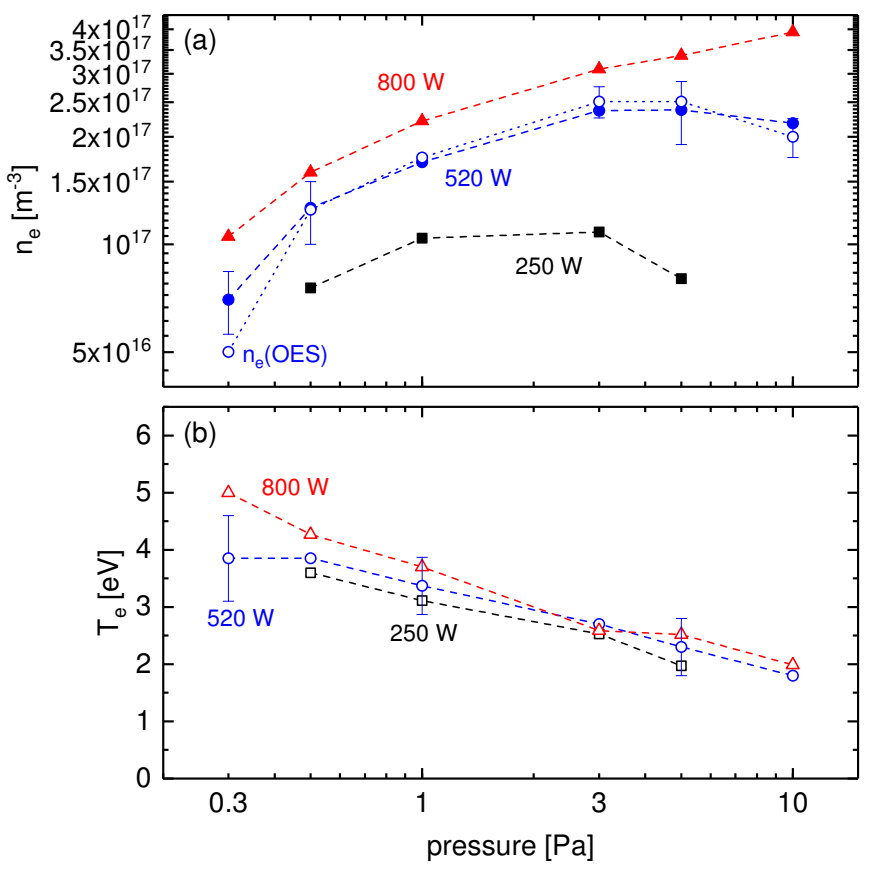

Figure 3. Plasma parameters of hydrogen discharges at varying pressure and delivered RF power. Depicted in (a) is the electron density obtained by probe measurements (full symbols) and via optical emission spectroscopy (open symbols). In (b), the electron temperature is shown, evaluated by optical emission spectroscopy and collisional radiative modelling. All presented values are averages along the cylinder axis.

In Figure 3 (b), the pressure dependent behaviour of the electron temperature determined via OES is shown. For all powers applied, it is characterized by a monotonous decrease from about 4 to $5 \mathrm{eV}$ at $0.3 \mathrm{~Pa}$ down to values slightly below $2 \mathrm{eV}$ at $10 \mathrm{~Pa}$, which corresponds to the expected behaviour in low pressure discharges. As expected furthermore, the influence of the applied power is not significant. All values of $T_{\mathrm{e}}$ are within the error margins for a fixed pressure, with the only exception at $0.3 \mathrm{~Pa}$. Generally, a small increase of $T_{\mathrm{e}}$ with increasing power is present, but almost exclusively within the measurement error.

In order to evaluate the plasma conductivity $\sigma_{\text {eff }}$, the effective collision frequency $\nu_{\text {eff }}$ is determined in the last step. Therefore, both $\nu_{\mathrm{en}}$ and $\nu_{\text {stoc }}$ are calculated based on the measured plasma parameters. In Figure 4, the resulting frequencies are presented at varying pressure for a delivered RF power of $520 \mathrm{~W}$. In addition to that, the value of the excitation frequency $\omega$ is shown, which is globally lower than all of the individually evaluated collision frequencies. This implicates according to Equation (5), that a lower collision frequency results in an increase of the plasma conductivity. The electron-neutral collision frequency is determined based on the momentum transfer cross section of electrons with molecular hydrogen provided by [16] and the assumption of a Maxwellian EEDF. The influence of hydrogen atoms on the collision frequency is neglected, as the density of molecules is a factor of 5 to 10 higher and the electron-impact rate coefficients of atomic and molecular hydrogen are well comparable in the electron temperature region of interest.

The evaluated collision frequency $\nu_{\mathrm{en}}$ displays an almost linear increase with rising pressure due to its proportionality to the neutral particle density. Slight deviations from a linear dependence are due to a change of the neutral density caused by a variation of the gas temperature, which rises steadily from $460 \mathrm{~K}$ at $0.3 \mathrm{~Pa}$ up to $700 \mathrm{~K}$ at $10 \mathrm{~Pa}$ due to the increased collision rate and energy transfer between electrons and neutrals. Furthermore, there is an implicit influence of the electron temperature on $\nu_{\mathrm{en}}$ via the rate coefficient $X_{\mathrm{en}}\left(T_{\mathrm{e}}\right)$. However, as there is only a weak variation of the electron momentum transfer cross section in hydrogen in the relevant energy region between 1 and $10 \mathrm{eV}$ [16], the impact of $T_{\mathrm{e}}$ on the electron collision frequency is almost negligible.

The stochastic collision frequency calculated according to Equation (3) displays only a weak variation over pressure. Comparing both $\nu_{\text {stoc }}$ and $\nu_{\text {en }}$, the expected dominance of ohmic heating at higher pressure is well reflected as $\nu_{\text {en }}$ exceeds $\nu_{\text {stoc }}$ by more than one order of magnitude. As the influence of stochastic heating is negligible in this region, the effective collision frequency is equivalent to $\nu_{\mathrm{en}}$. Towards lower pressures of $p \leq 1 \mathrm{~Pa}$ however, the influence of stochastic heating is significant. At the low pressure limit of $0.3 \mathrm{~Pa}$, the collision frequencies are of the same order of magnitude. Consecutively, the effective collision frequency deviates from $\nu_{\mathrm{en}}$, which confirms that stochastic heating indeed has to be considered in hydrogen discharges at low pressure. The totally delivered RF power has only a minor influence on the evaluated collision frequencies. Exemplarily, the effective collision frequency at a delivered $\mathrm{RF}$ power of $250 \mathrm{~W}$ is depicted as well in Figure 5. The relative behaviour is virtually the same as for $520 \mathrm{~W}$. The absolute frequency is slightly but systematically higher, which is mainly due to a slightly higher neutral density caused by a lower gas temperature. For higher $\mathrm{RF}$ power, the correlation is analogous and leads to an effective reduction of $\nu_{\text {eff }}$.

Taking into account the plasma parameters discussed above, the real part of the complex plasma 


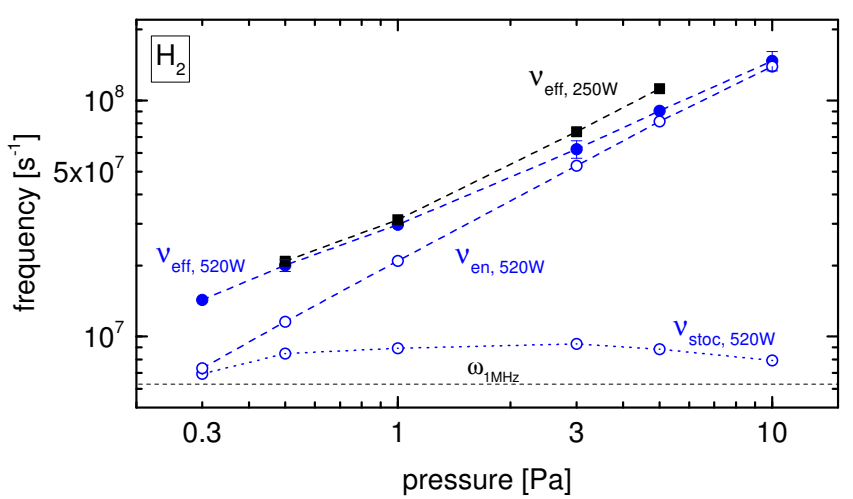

Figure 4. Pressure and power dependency of the electron momentum transfer frequencies in hydrogen. Depicted is the collision frequency of electrons with neutrals $\left(\nu_{\mathrm{en}}\right)$, the frequency of the electron momentum change due to stochastic non-collisional heating $\left(\nu_{\text {stoch }}\right)$ and the effective sum of both components $\left(\nu_{\text {eff }}\right)$.

conductivity is determined according to Equation (5). $\sigma_{\text {eff }}$ is shown in Figure 5 at varying pressure and RF power. With an increase of the delivered RF power, the conductivity increases systematically. For all three RF powers applied, an analogous behaviour with respect to the pressure is obtained: $\sigma_{\text {eff }}$ reaches a maximum between 0.5 and $1 \mathrm{~Pa}$ and decreases for both higher and lower pressures, with the exception of $250 \mathrm{~W}$, where no stable plasma operation is possible below $0.5 \mathrm{~Pa}$. In general, the obtained picture resembles well the relative behaviour of the RF power transfer efficiency presented in Figure 2, which allows for a detailed comparison between $\eta$ and $\sigma_{\text {eff }}$ and an evaluation of the influence of the individual plasma parameters which determine the power absorption by the plasma.

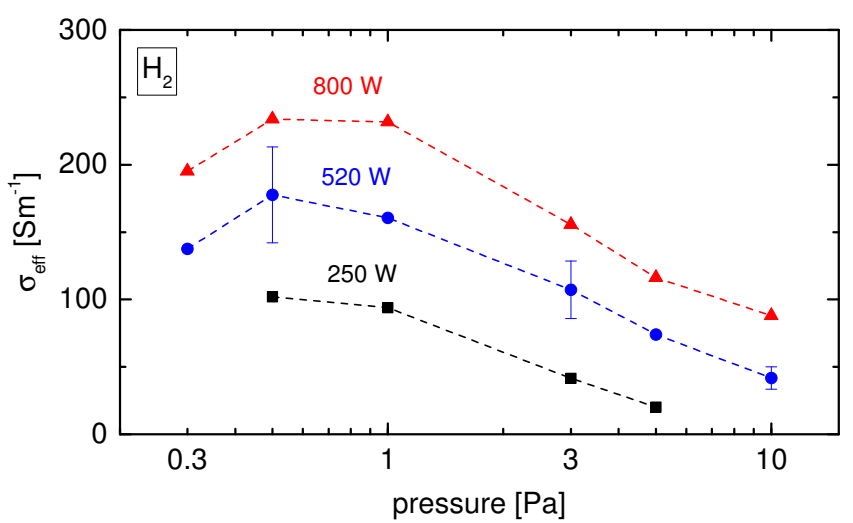

Figure 5. Real part of the complex plasma conductivity at varying pressure and totally delivered $\mathrm{RF}$ power in hydrogen.

First, the increase of $\eta$ as well as $\sigma_{\text {eff }}$ for higher $P_{\mathrm{RF}, \text { del }}$ is mainly caused by the increasing electron density. Furthermore, the observed slight decrease of the effective collision frequency with increasing power leads to an additional increase of the plasma conductivity, as this corresponds to an increase of the fraction $\nu_{\text {eff }} /\left(\omega^{2}+\nu_{\text {eff }}^{2}\right)$ in the present case, where $\omega<$ $\nu_{\text {eff }}$ is valid globally. Secondly, the characteristically peaked pressure dependence of the plasma conductivity is generally comparable to the $\mathrm{RF}$ power transfer efficiency as well. It is caused by an interplay of the electron density and the effective collision frequency. At low pressure, $\nu_{\text {eff }}$ is at its minimum near the applied frequency $\omega$, which increases the plasma conductivity. Then again, the low value of $n_{\mathrm{e}}$ significantly reduces the conductivity and consecutively the ability of the plasma to absorb power. Towards higher pressure the situation inverts: while the electron density increases, proportionally enhancing the plasma conductivity, the steady increase of $\nu_{\text {eff }}$ simultaneously reduces $\sigma_{\text {eff }}$. Consequently, both $\sigma_{\text {eff }}$ as well a $\eta$ describe the observed peak at intermediate pressure and decrease significantly at higher pressure, where the influence of the rising collisions effectively reduces the plasma's capability to absorb the delivered RF power.

Nevertheless the general dependencies of $\eta$ on gas pressure and RF power appear to be well resembled by the presented analytical consideration, a direct comparison of the Figures 2 and 5 also reveals that the position of the pressure dependent peak of $\sigma_{\text {eff }}$ is shifted to slightly lower pressure, in relation to the trend of the RF power transfer efficiency. The cause of this local deviation cannot be unambiguously identified, but several issues can be considered.

First, it is known that the EEDF in inductive discharges may deviate from a Maxwellian distribution, particularly at low pressures. Since a Maxwellian EEDF has been globally considered for the evaluation of the electron-neutral collision frequency, this could also affect the evaluated plasma conductivity, particularly in the low pressure region. However, the influence of the electron temperature on $\nu_{\mathrm{en}}$ in the temperature region of interest is weak, as already discussed above. Therefore also the effect of a non-Maxwellian EEDF on the plasma conductivity is considered to be small, and most certainly not prominent enough to serve as a possible explanation for the observed deviation. Second, the observed behaviour may be caused by the fact that this zero dimensional investigation of the power absorption does not account for changes of the spatial profiles of the involved plasma parameters with pressure and power and the associated influence of the electric field $\tilde{\mathbf{E}}$ in the RF sheath. For a spatially resolved treatment of the heating process, numerical modelling approaches are required, however. In addition, the contradictions of $\sigma_{\text {eff }}$ and $\eta$ at low pressure may also be a result of a limited validity of the considered descrip- 
tion of stochastic heating. As mentioned already in section 2 , the ion plasma frequency of the investigated discharges is higher than the excitation frequency, in contrast to the assumptions made in [10]. To what extend this deteriorates the description of stochastic heating at the present conditions cannot be quantitatively assessed at the moment, though. Further investigations, e.g. at a higher excitation frequency, could provide useful additional insight.

\subsection{Relevance of atoms in molecular discharges: comparison of $\mathrm{H}_{2}$ and $\mathrm{D}_{2}$}

As it is shown in the previous section, the relative behaviour of the RF power absorption of hydrogen discharges at varying pressure and power is in general well comparable to the results obtained for noble gas discharges. Due to dissociation processes however, also atoms are present in molecular discharges. If the dissociation is high enough, atoms are in the same order of magnitude as molecules and have to be considered when the RF power absorption process of these discharges is analysed. In order to investigate the specific influence of atoms on the RF power absorption, a direct comparison of hydrogen and deuterium discharges is performed.

This approach is suitable due to the uniqueness that in low-pressure deuterium and hydrogen discharges many of the crucial elastic and inelastic collision processes are similar, as the difference in mass has generally no impact on the electronic potential energy curves of the molecules, which leads to typically comparable plasma parameters at the same operational parameters. However, significant isotopic effects are present for the vibrational and rotational levels, which particularly influences the processes leading to molecular dissociation. A specifically comparative review regarding the electron-impact cross sections of the hydrogen molecule and its isotopomers is for example provided by Yoon $[16,17]$. As it is shown therein by referencing computational cross section data [26, 27], the dissociation rate of the hydrogen isotopomers near the dissociation energy threshold (which is in the energy region to be considered in low pressure $\mathrm{H}_{2} / \mathrm{D}_{2}$ discharges) is increasing with increasing molecular mass. Consequently, the dissociation of molecules is expected to be considerably higher in deuterium than in hydrogen.

Experimentally, the influence of the molecular dissociation can be specifically investigated by evaluating the ratio $n_{\mathrm{H}} / n_{\mathrm{H}_{2}}$ of the atomic to the molecular density in hydrogen - and analogously $n_{\mathrm{D}} / n_{\mathrm{D}_{2}}$ in deuterium - via optical emission spectroscopy. For the present case, the corresponding values of this ratio at varying pressure are shown in Figure 6 (a) for both hydrogen and deuterium, exemplarily for a delivered RF power of $250 \mathrm{~W}$. Both density ratios describe a comparable relative dependence on the pressure with a maximum at $1 \mathrm{~Pa}$ and decrease towards lower and higher pressure. There is indeed a significant isotopic deviation, as $n_{\mathrm{D}} / n_{\mathrm{D}_{2}}$, which reaches values between $30 \%$ and $45 \%$, globally exceeds $n_{\mathrm{H}} / n_{\mathrm{H}_{2}}$ by an approximate factor of 2. Analogously, an isotopic difference of the same order of magnitude is present also for applied RF powers of 520 and $800 \mathrm{~W}$. Consequently, the atomic density in $\mathrm{D}_{2}$ plasmas is significantly higher than in $\mathrm{H}_{2}$ at the same operational parameters, as expected from literature. Furthermore, the absolute densities of atoms are in the same order as the density of the molecules, which allows for a dedicated analysis of the influence of atoms on the RF power absorption process.

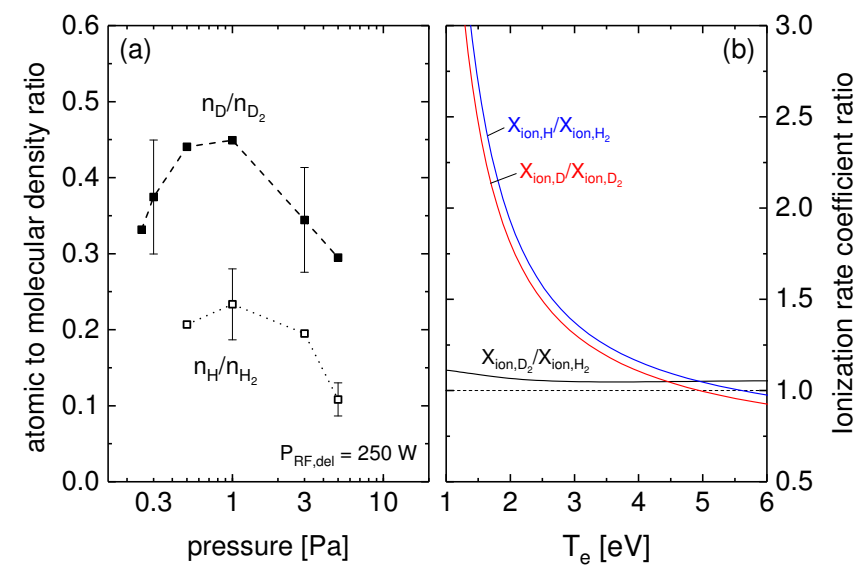

Figure 6. (a) ratio of the atomic to the molecular density at varying pressure at $P_{\mathrm{RF}, \text { del }}=250 \mathrm{~W}$ in hydrogen and deuterium. (b) ratio of the electron impact ionization rate coefficients of hydrogen and deuterium atoms and molecules in the electron temperature region of interest between 1 and $6 \mathrm{eV}$, calculated based on cross section data by $[16,28]$ and by assuming a Maxwellian EEDF. The horizontal dotted line indicates unity.

As already discussed in section 4.1, the influence of the atoms on the total electron-neutral collision frequency is negligible in the present case. In opposition to that, a significant difference between atoms and molecules can be found for the electronimpact ionization due to the lower ionization energy of hydrogen atoms $(13.60 \mathrm{eV})$ compared to molecules $(15.60 \mathrm{eV})$. In order to quantify this difference, the rate coefficients $X_{\mathrm{ion}}\left(T_{\mathrm{e}}\right)$ of electron-impact ionization are evaluated assuming a Maxwellian EEDF based on total electron-impact ionization cross sections of both the molecules $\mathrm{H}_{2}$ and $\mathrm{D}_{2}[16,17]$ as well as the atoms $\mathrm{H}$ and $\mathrm{D}$ (which are considered identical)[28]. Due to the different threshold energies, the obtained rate coefficients display a significant deviation at low electron temperatures, which is illustrated in Figure 6 (b). Presented are the rate coefficient ratios of 
$X_{\text {ion }, \mathrm{H}} / X_{\text {ion }, \mathrm{H}_{2}}, X_{\text {ion, } \mathrm{D}} / X_{\text {ion, } \mathrm{D}_{2}}$ and $X_{\text {ion, } \mathrm{D}_{2}} / X_{\text {ion, } \mathrm{H}_{2}}$ in the electron temperature range between 1 and $6 \mathrm{eV}$. For both deuterium and hydrogen, the rate coefficients of electron-impact ionization of the atoms are generally higher than those of the molecules at electron temperatures below $5 \mathrm{eV}$. This difference increases strongly with decreasing temperature. For electron temperatures above $5 \mathrm{eV}$ the situation is inverse, as the influence of the lower ionization threshold of atoms decreases and additional ionization reactions possible only in molecules increasingly contribute. As depicted by the ratio $X_{\mathrm{ion}, \mathrm{D}_{2}} / X_{\mathrm{ion}, \mathrm{H}_{2}}$, the ionization rate coefficient of the $\mathrm{D}_{2}$ molecule is systematically about $5 \%$ higher than the $\mathrm{H}_{2}$ molecule, which is due to the same isotopic effects influencing the dissociation.
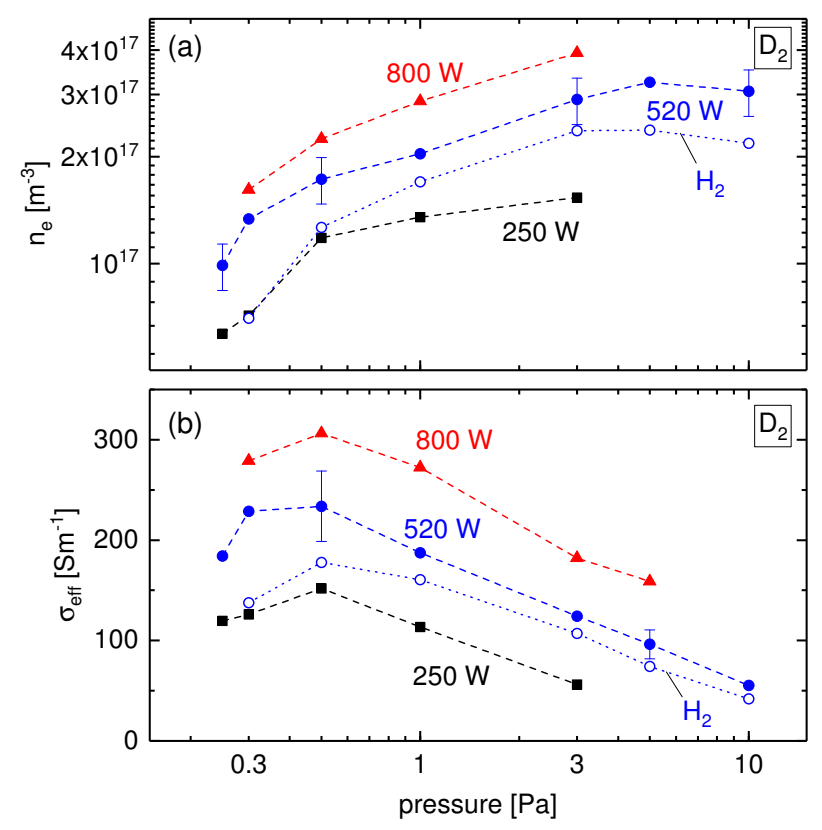

Figure 7. (a) electron density in deuterium at varying pressure and delivered RF power. (b) plasma conductivity in deuterium. The results obtained in hydrogen at $520 \mathrm{~W}$ are included for a comparison, respectively.

Consequently, a higher ionisation rate in $\mathrm{D}_{2}$ is expected compared to hydrogen, to a minor extend because $\mathrm{D}_{2}$ molecules are slightly easier ionized than $\mathrm{H}_{2}$ molecules, but mainly due to the increased atomic fraction. Experimentally, this is well reflected by the measured electron density in deuterium discharges which is depicted in Figure 7 (a). Generally, $n_{\mathrm{e}}$ is characterized by the same relative dependencies on the operational parameters $p$ and $P_{\mathrm{RF} \text {,del }}$ that are observed in hydrogen. However, as illustrated by the direct comparison with the exemplary included density in $\mathrm{H}_{2}$ at $520 \mathrm{~W}$, the absolute electron densities in deuterium are systematically higher. The values generally exceed the ones obtained in hydrogen by roughly $50 \%$, which further increases at low pressure.

Consecutively, the power absorption mechanism in deuterium is analysed analogous to hydrogen in section 4.1 by evaluating the plasma conductivity $\sigma_{\text {eff }}$ according to Equation (5). The general behaviour of the further required parameters $T_{\mathrm{e}}$ and $\nu_{\mathrm{eff}}$ are found to be well comparable to the situation observed in $\mathrm{H}_{2}$. Firstly, within the error margins the electron temperature in deuterium is similar to the one in hydrogen which is shown in Figure 3, being mainly dominated by the charactersitic decrease with pressure. Secondly, the electron collision frequency is also not displaying any significant isotopic differences and remains virtually the same as in hydrogen. This is caused by the counteracting of a slightly elevated electron-neutral collision cross section [17] and a simultaneously reduced gas temperature (roughly between 50 to $100 \mathrm{~K}$ due to the higher mass).

The resulting values of $\sigma_{\text {eff }}$ are shown in Figure 7 (b), where they are directly compared to the values obtained in $\mathrm{H}_{2}$ at $520 \mathrm{~W}$. As expected, generally the same relative dependence of $\sigma_{\text {eff }}$ on the the pressure and the power is observed for deuterium and hydrogen, as all considered input parameters depict comparable trends as well. However, the absolute value of the conductivity is systematically higher in $\mathrm{D}_{2}$, which is directly caused by its proportionality to $n_{\mathrm{e}}$.

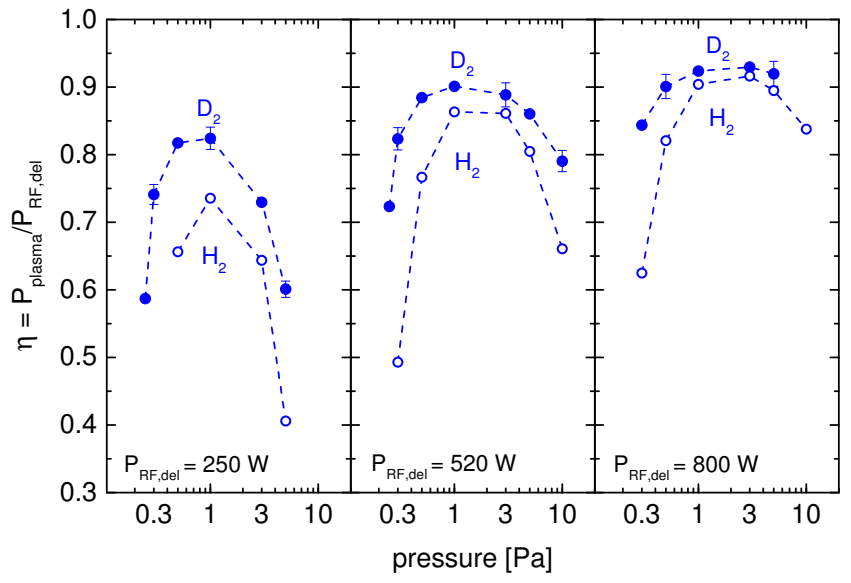

Figure 8. RF power transfer efficiency $\eta$ in deuterium at varying pressure and at three different values of totally delivered $\mathrm{RF}$ power; included for comparison are the results obtained in hydrogen.

The isotopic difference of $\sigma_{\text {eff }}$ results in an increased capability of the deuterium plasma to absorb the provided RF power, which is in excellent agreement with the measurement of the RF power transfer efficiency shown in Figure 8 . While the observed relative dependencies of $\eta$ on the operational 
parameters $p$ and $P_{\mathrm{RF}, \text { del }}$ are quite similar in $\mathrm{D}_{2}$ and $\mathrm{H}_{2}$, a systematically higher power absorption is measured in deuterium. Whereas at higher powers and in the pressure peak region this isotopic difference is only of a few percent, it is significantly evident at higher and particularly at lower pressures. At the intermediate $\mathrm{RF}$ power of $520 \mathrm{~W}$ for example, the efficiency in $\mathrm{D}_{2}$ at $0.3 \mathrm{~Pa}$ is still above $80 \%$, while it drops below $50 \%$ in the case of $\mathrm{H}_{2}$. Due to the increased efficiency in deuterium, stable inductive plasma operation down to $0.25 \mathrm{~Pa}$ becomes possible. The particularly increased efficiency in $\mathrm{D}_{2}$ at lower pressures very well resembles the behaviour of $\sigma_{\text {eff }}$ shown in Figure 7, where the difference between hydrogen and deuterium is also most prominent at low pressures of $p<1 \mathrm{~Pa}$.

In summary, the presented results illustrate that the atomic fraction in $\mathrm{H}_{2} / \mathrm{D}_{2}$ low pressure discharges has to be considered when the RF heating and power transfer mechanism is investigated: A higher dissociation degree leads to a significant increase of the ionization rate in the present electron temperature range, effectively increasing the electron density and thereby the capability of the plasma to absorb the provided RF power.

\section{Conclusion}

The RF power transfer efficiency $\eta$ of inductively heated hydrogen and deuterium discharges has been investigated at an excitation frequency of $1 \mathrm{MHz}$. For both isotopomers, a comparable dependence on the varied operational parameters is observed: while $\eta$ globally increases with increasing RF power, a local maximum between 1 and $3 \mathrm{~Pa}$ is observed when the gas pressure is varied. The observed dependencies of $\eta$ on the operational parameters are found to be well explained by an analytical approach considering the power absorption of the plasma by evaluating the RF plasma conductivity based on the measured plasma parameters. The direct comparison of $\mathrm{H}_{2}$ and $\mathrm{D}_{2}$ reveals the importance to consider the presence of different neutral species within molecular discharges. Due to the higher molecular dissociation, the atomic density in deuterium discharges is systematically higher than in hydrogen. As the atoms are more efficiently ionized in the present electron temperature region between 1 and $5 \mathrm{eV}$ than the respective molecules due to the lower threshold energy of electron-impact ionization, this leads to a significantly elevated electron density in $\mathrm{D}_{2}$ compared to $\mathrm{H}_{2}$, and consecutively to a globally higher RF power transfer efficiency.

\section{Acknowledgments}

This work has been carried out within the framework of the EUROfusion Consortium and has received funding from the Euratom research and training programme 2014-2018 under grant agreement No 633053. The views and opinions expressed herein do not necessarily reflect those of the European Commission.

The authors would like to thank the Deutsche Forschungsgemeinschaft (DFG) for their support within the project BR 4904/1-1.

\section{References}

[1] J. Hopwood. Plasma Sources Science and Technology, 1:109, 1992.

[2] M. A. Lieberman and A. J. Lichtenberg. Principles of Plasma Discharges and Materials Processing, 2nd edition. John Wiley \& Sons, Hoboken, New Jersey, 2005.

[3] P. Chabert and N. Braithwaite. Physics of RadioFrequency Plasmas. Cambridge, 2011.

[4] R. B. Piejak, V. A. Godyak, and B. M. Alexandrovich. Plasma Sources Science and Technology, 1:179, 1992.

[5] J. Hopwood. Plasma Sources Sci. Technol., 3:460-464, 1994.

[6] K. Suzuki, K. Nakamura, H. Ohkubo, and H. Sugai. Plasma Sources Science and Technology, 7:13, 1998.

[7] V. A. Godyak, R. B. Piejak, and B. M. Alexandrovich. Journal of Applied Physics, 85:703-712, 1999.

[8] E. A. Kralkina. Physics-Uspekhi, 51:493-512, 2008.

[9] M. M. Turner. Phys. Rev. Lett., 71:1844-1847, 1993.

[10] V. Vahedi et al. Journal of Applied Physics, 78:1446-1458, 1995.

[11] M. A. Lieberman and V. A. Godyak. IEEE Transactions on Plasma Science, 26:955-986, 1998.

[12] J. Lettry et al. Rev. Sci. Instrum., 87:02B139, 2016.

[13] R. Hemsworth et al. Nuclear Fusion, 49:045006, 2009.

[14] C. M. Horwitz. Journal of Vacuum Science \&3 Technology A, 1:1795-1800, 1983.

[15] V. A. Godyak and R. B. Piejak. Journal of Vacuum Science Es Technology A, 8:3833-3837, 1990.

[16] J.-S. Yoon et al. Journal of Physical and Chemical Reference Data, 37:913-931, 2008.

[17] J.-S. Yoon et al. Reports on Progress in Physics, 73(11):116401, 2010.

[18] V. Godyak. Physics of Plasmas, 12:055501, 2005.

[19] D. Wünderlich, S. Dietrich, and U. Fantz. Journal of Quantitative Spectroscopy and Radiative Transfer, 110:62-71, 2009.

[20] D. Wünderlich and U. Fantz. Atoms, 4:26, 2016.

[21] U. Fantz et al. Nuclear Fusion, 46:297-306, 2006.

[22] S. Briefi et al. Plasma Sources Science and Technology, 25(3):035015, 2016.

[23] E. O. Johnson and L. Malter. Phys. Rev., 80:58-68, 1950.

[24] F. F. Chen, J. D. Evans and W. Zawalski. Plasma Diagnostic Techniques. Academic Press, 1965.

[25] E. A. Kralkina et al. Plasma Sources Science and Technology, 25:015016, 2016.

[26] C. S. Trevisan and J. Tennyson. Plasma Physics and Controlled Fusion, 44:1263, 2002

[27] C. S. Trevisan and J. Tennyson. Plasma Physics and Controlled Fusion, 44:2217, 2002.

[28] M. B. Shah, D. S. Elliott, and H. B. Gilbody. Journal of Physics B: Atomic and Molecular Physics, 20:3501, 1987. 